\title{
A SUPERLINEARLY CONVERGENT METHOD FOR A CLASS OF COMPLEMENTARITY PROBLEMS WITH NON-LIPSCHITZIAN FUNCTIONS*
}

\author{
GUANGLU ZHOU ${ }^{\dagger}$, LOUIS CACCETTA ${ }^{\ddagger}$, AND KOK LAY TEO ${ }^{\S}$
}

\begin{abstract}
We consider a class of complementarity problems involving functions which are not Lipschitz continuous. In this paper we reformulate this class of non-Lipschitzian complementarity problems into a Lipschitzian complementarity problem. Then we propose an inexact smoothing Newton method to solve this Lipschitzian complementarity problem. We prove that our proposed method converges quadratically and globally under a mild condition. Numerical results show that this method is promising. This method can solve these kinds of complementarity problems with one million variables in reasonable time on a PC with 1 GB of RAM.
\end{abstract}

Key words. complementarity problems, non-Lipschitzian continuity, nonsmooth equations, smoothing Newton method, superlinear convergence

AMS subject classifications. 90C $33,90 \mathrm{C} 30,65 \mathrm{H} 10$

DOI. $10.1137 / 080726690$

1. Introduction. Let $\Re_{+}^{n}=\left\{x \in \Re^{n}: x \geq 0\right\}$, and define $F: \Re_{+}^{n} \rightarrow \Re^{n}$ by

$$
F(x)=M x+B x^{p}+q,
$$

where $M$ is an $n \times n$ matrix, $B$ is an $n \times n$ diagonal matrix with positive diagonals, $x^{p}=\left[x_{1}^{p}, \ldots, x_{n}^{p}\right]^{T}, p \in(0,1)$, and $q$ is a vector in $\Re^{n}$. Because $p \in(0,1)$, the function $F$ is not Lipschitz continuous at a vector which has zero component. In this paper we consider the following nonlinear complementarity problem (NCP) and denote it by $\operatorname{NCP}(\mathrm{F})$. Find $x \in \Re_{+}^{n}$ such that

$$
x \geq 0, \quad F(x) \geq 0, \quad x^{T} F(x)=0 .
$$

Such a non-Lipschitzian NCP arises from the reaction and diffusion problems [1, 4], which can be modeled as free boundary problems.

Example 1. [1,4] Let $\Omega$ be a bounded open set in $\Re^{2}$ with Lipschitz boundary $\partial \Omega$. Given two positive numbers $\lambda$ and $p \in(0,1)$, consider the following free boundary problem:

$$
\begin{aligned}
& -\triangle u+\lambda u^{p}=0 \quad \text { in } \Omega_{+}, \\
& u=0 \text { in } \Omega_{0} \text {, } \\
& u=|\nabla u|=0 \quad \text { on } \Gamma \text {, } \\
& u=1 \quad \text { on } \partial \Omega \text {, }
\end{aligned}
$$

${ }^{*}$ Received by the editors June 9, 2008; accepted for publication (in revised form) October 25, 2009; published electronically February 19, 2010. This work was supported by the Australian Research Council and the National Natural Science Foundation of China (grant 10771120).

http://www.siam.org/journals/siopt/20-4/72669.html

${ }^{\dagger}$ Corresponding author. Western Australian Centre of Excellence in Industrial Optimisation (WACEIO), Department of Mathematics and Statistics, Curtin University of Technology, Perth, WA 6845, Australia (G.Zhou@curtin.edu.au).

${ }^{\ddagger}$ Western Australian Centre of Excellence in Industrial Optimisation (WACEIO), Department of Mathematics and Statistics, Curtin University of Technology, Perth, WA 6845, Australia (caccetta@ maths.curtin.edu.au).

$\S$ Department of Mathematics and Statistics, Curtin University of Technology, Perth, WA 6845, Australia (K.L.Teo@curtin.edu.au). 
where $\Omega_{+}=\{z \in \Omega: u(z)>0\}, \Omega_{0}=\{z \in \Omega: u(z)=0\}$, and $\Gamma=\partial \Omega_{0}=\partial \Omega_{+} \cap \Omega$ are unknown, and $\triangle u=\frac{\partial^{2} u}{\partial z_{1}^{2}}+\frac{\partial^{2} u}{\partial z_{2}^{2}}$. Using finite element approximation or finite difference approximation, we obtain an NCP (1.1). Note that in (1.1), $F$ is defined only on $\Re_{+}^{n}$. For the NCP obtained from Example 1, $F$ has no definition on $\Re^{n} \backslash \Re_{+}^{n}$.

Over the last decade, a number of methods for solving the NCP have been developed (see [9, Chapters 9 and 11]). However, most of these efficient methods require the involved function $F$ to be Lipschitz continuous. For instance, smoothing Newton methods $[6,14]$ and semismooth Newton methods [8] assume that $F$ is continuously differentiable. For these methods, in order to get the Q-quadratic convergence property, it is assumed that $F^{\prime}$ is Lipschitz continuous. Without the Lipschitzian continuity of $F$, it seems hard to find a fast convergent method to solve the $\operatorname{NCP}(\mathrm{F})$.

Recently, Chen [5] presented a smoothing Newton method for solving the following system of equations with non-Lipschitzian functions by reformulating it as a system of equations with locally Lipschitzian functions.

$$
S(x):=M x+B f(x)+q=0,
$$

where for $i=1,2, \ldots, n$,

$$
f_{i}(x)=f_{i}\left(x_{i}\right)= \begin{cases}x_{i}^{p}, & x_{i} \geq 0 \\ 0, & x_{i}<0\end{cases}
$$

Under the condition that $M$ is a symmetric positive definite matrix, it is proved that the smoothing Newton method [5] converges superlinearly and globally. For a general class of non-Lipschitzian complementarity problems, a late research work was given by Alefeld and Chen [1], in which a regularized projection method has been proposed for solving this general class of non-Lipschitzian complementarity problems. This projection method has global and linear convergence properties under the condition that $M$ is an $H$-matrix with positive diagonals.

An $n \times n$ matrix $A=\left(a_{i j}\right)$ is called a positive definite matrix if for any $x \in \Re^{n}$ with $x \neq 0, x^{T} A x>0$. The matrix $A$ is called a $P$-matrix if, for every $x \in \Re^{n}$ with $x \neq 0$, it holds that

$$
\max _{i} x_{i}[A x]_{i}>0 .
$$

The matrix $A$ is called a $P_{0}$-matrix if, for every $x \in \Re^{n}$ with $x \neq 0$, there is an index $i_{0}=i_{0}(x)$ with

$$
x_{i_{0}} \neq 0 \quad \text { and } \quad x_{i_{0}}[A x]_{i_{0}} \geq 0 .
$$

Clearly a positive definite matrix is a $P$-matrix, and a $P$-matrix is a $P_{0}$-matrix. $A$ is called an $M$-matrix [12] if (i) $a_{i i}>0$ for $i=1,2, \ldots, n$, (ii) $a_{i j} \leq 0(i \neq j$ ) for $i, j=1,2, \ldots, n$, and (iii) $A$ is nonsingular and $A^{-1} \geq 0$. Here $A^{-1} \geq 0$ means that each entry of $A^{-1}$ is nonnegative. $A$ is called an $H$-matrix if its comparison matrix $\tilde{A}=\left(\tilde{a}_{i j}\right)$ is an $M$-matrix, where

$$
\tilde{a}_{i j}=\left\{\begin{aligned}
\left|a_{i j}\right|, & i=j, \\
-\left|a_{i j}\right|, & i \neq j, \quad i, j=1,2, \ldots, n .
\end{aligned}\right.
$$

An $H$-matrix with positive diagonals is a $P$-matrix.

The non-Lipschitzian NCP (1.1) and the non-Lipschitzian system (1.2) have a number of applications on some very important problems in physics and finance, such 
as the reaction-diffusion problems [3, 4], the nonlinear parabolic complementarity problem [21], and European and American option valuation $[2,22]$. In order to find a numerical solution for these problems, we can use a discretization method such as the central difference, the piecewise linear finite element, or a finite volume method $[21,22]$ to reduce these problems into (1.1) or (1.2). Then a numerical solution of these problems is obtained by solving (1.1) or (1.2). However, the resulting problem (1.1) or (1.2) is usually very large.

In this paper we propose a superlinearly convergent method to solve the $\mathrm{NCP}(\mathrm{F})$ (1.1). In section 2 we give some equivalent formulations for the $\mathrm{NCP}(\mathrm{F})$. In particular, by using the techniques in [5], we reformulate the $\mathrm{NCP}(\mathrm{F})$ as a nonlinear complementarity problem involving functions which are Lipschitz continuous. This is a crucial step in the development of a Newton-type method with superlinear convergence property for the $\mathrm{NCP}(\mathrm{F})$. In addition, under the condition that $M$ is a $P_{0}$-matrix, we show that the $\operatorname{NCP}(\mathrm{F})$ has a unique solution and the reformulated system enjoys a desirable nonsingularity property. In section 3 we present a superlinearly convergent algorithm for solving the $\mathrm{NCP}(\mathrm{F})$, which is an inexact version of the smoothing methods proposed in [14]. We show that our proposed method has global and quadratic convergence properties under the condition that $M$ is a $P_{0}$-matrix. This condition is weaker than the ones used in $[1,5]$. Our numerical results reported in section 4 show that our proposed method can produce an approximate solution with high accuracy for large-scale problems. We conclude the paper with some remarks in section 5 .

We conclude this section with some notation and terminology. For a continuously differentiable function $D: \Re^{n} \rightarrow \Re^{n}$, we denote the Jacobian of $D$ at $x \in \Re^{n}$ by $D^{\prime}(x)$. Let $D: \Re^{n} \rightarrow \Re^{n}$ be a locally Lipschitzian vector function. By Rademacher's theorem, $D$ is differentiable almost everywhere. Let $U_{D}$ denote the set of points where $D$ is differentiable. Then the B-subdifferential of $D$ at $x \in \Re^{n}$ is defined by

$$
\partial_{B} D(x)=\left\{\lim _{\substack{x^{k} \rightarrow x \\ x^{k} \in U_{D}}} D^{\prime}\left(x^{k}\right)\right\},
$$

while Clarke's generalized Jacobian of $D$ at $x$ is defined by

$$
\partial D(x)=\operatorname{conv}_{B} D(x)
$$

(see $[7,15]) . D$ is called semismooth at $x$ if $D$ is directionally differentiable at $x$ and for all $V \in \partial D(x+h)$ and $h \rightarrow 0$,

$$
D^{\prime}(x ; h)=V h+o(\|h\|)
$$

$D$ is called strongly semismooth at $x$ if $D$ is semismooth at $x$ and for all $V \in \partial D(x+h)$ and $h \rightarrow 0$,

$$
D^{\prime}(x ; h)=V h+O\left(\|h\|^{2}\right) .
$$

For $x \in \Re^{n}$, the 2 -norm is denoted by $\|x\|$. For a convex set $X \subset \Re^{n}, \Pi_{X}(\cdot)$ is the Euclidean projection operator onto $X$, which is defined as follows:

$$
\Pi_{X}(x)=\underset{y \in X}{\operatorname{argmin}}\|x-y\|, \quad x \in \Re^{n} .
$$

For a vector $x \in \Re^{n}, \operatorname{diag}(x)$ denotes the $n \times n$ diagonal matrix generated by $x$. For an $n \times n$ diagonal matrix $B, B_{i}$ denotes the $i$ th diagonal of $B$. Let $\Re_{+}=\{t \in \Re: t \geq 0\}$ and $\Re_{++}=\{t \in \Re: t>0\}$. Finally, we use $t \downarrow 0^{+}$to denote the case that a positive scalar $t$ tends to 0 . 
2. Some equivalent formulations. In this section, we give some equivalent formulations for the $\mathrm{NCP}(\mathrm{F})$. In particular, we reformulate the $\mathrm{NCP}(\mathrm{F})$ as an $\mathrm{NCP}$ involving functions which are Lipschitz continuous, which is a vital step in the development of a superlinearly convergent method for the $\operatorname{NCP}(\mathrm{F})$. The idea of this reformulation is same as the one in [5]. It is proved that the smoothing Newton method [5] converges superlinearly and globally if $M$ is a symmetric positive definite matrix. In this paper, we will show our proposed method converges quadratically and globally under the condition that $M$ is a $P_{0}$-matrix, which is a nice property of our method.

For a nonnegative vector $x \in \Re^{n}$ and $p \in(0,1)$, let $y=\left[x_{1}^{p}, x_{2}^{p}, \ldots, x_{n}^{p}\right]^{T}$ and $y^{\frac{1}{p}}=\left[y_{1}^{\frac{1}{p}}, \ldots, y_{n}^{\frac{1}{p}}\right]^{T}$. Define $W: \Re_{+}^{n} \rightarrow \Re^{n}$ by

$$
W(y)=M y^{\frac{1}{p}}+B y+q .
$$

Then the $\mathrm{NCP}(\mathrm{F})$ becomes the following complementarity problem:

$$
y^{\frac{1}{p}} \geq 0, \quad W(y) \geq 0, \quad\left(y^{\frac{1}{p}}\right)^{T} W(y)=0 .
$$

Let $\mathrm{NCP}(\mathrm{W})$ be the following $\mathrm{NCP}$ :

$$
y \geq 0, \quad W(y) \geq 0, \quad y^{T} W(y)=0 .
$$

Clearly, (2.2) and (2.3) are two equivalent problems. Hence, we have the following proposition.

Proposition 2.1. $N C P(W)$ is a Lipschitzian NCP. If $y$ is a solution of the $N C P(W)$, then $x=y^{\frac{1}{p}}$ is a solution of the $N C P(F)$. Conversely, if $x$ is a solution of the $N C P(F)$, then $y=\left[x_{1}^{p}, x_{2}^{p}, \ldots, x_{n}^{p}\right]^{T}$ is a solution of the $N C P(W)$.

For the $\mathrm{NCP}(\mathrm{W})$, we have the following proposition. The proof of this proposition will be given later.

Proposition 2.2. If $M$ is a $P_{0}$-matrix, then the $N C P(W)$ has a unique solution.

From Propositions 2.1 and 2.2, we obtain the following proposition.

Proposition 2.3. If $M$ is a $P_{0}$-matrix, then the non-Lipschitzian $N C P(F)(1.1)$ has a unique solution.

Let $X=\Re_{+}^{n}$. In order to prove that Proposition 2.2 holds, we need the following results.

Lemma 2.1. If $M$ is a $P_{0}$-matrix, then the function $W$ is a $P$-function on $X$; i.e., for any $x, y \in X$ with $x \neq y$, it holds that

$$
\max _{1 \leq i \leq n}\left(x_{i}-y_{i}\right)\left[W_{i}(x)-W_{i}(y)\right]>0 .
$$

Proof. For any $x, y \in X$ with $x \neq y$, since $M$ is a $P_{0}$-matrix, there exists an index $i$ such that

$$
x_{i} \neq y_{i} \quad \text { and } \quad\left(x_{i}^{1 / p}-y_{i}^{1 / p}\right)\left[M\left(x^{1 / p}-y^{1 / p}\right)\right]_{i} \geq 0 .
$$

For two nonnegative numbers $x_{i}$ and $y_{i}$ with $x_{i} \neq y_{i}$, we have $x_{i}^{1 / p}-y_{i}^{1 / p}>0(<0)$ if and only if $x_{i}-y_{i}>(<0)$. Thus,

$$
\left(x_{i}-y_{i}\right)\left[M\left(x^{1 / p}-y^{1 / p}\right)\right]_{i} \geq 0 .
$$


Hence,

$$
\begin{aligned}
\left(x_{i}-y_{i}\right)[W(x)-W(y)]_{i} & =\left(x_{i}-y_{i}\right)\left[M\left(x^{1 / p}-y^{1 / p}\right)\right]_{i}+B_{i}\left(x_{i}-y_{i}\right)^{2} \\
& \geq B_{i}\left(x_{i}-y_{i}\right)^{2}>0 .
\end{aligned}
$$

Therefore, we have

$$
\max _{1 \leq i \leq n}\left(x_{i}-y_{i}\right)[W(x)-W(y)]_{i}>0 .
$$

This completes the proof.

Lemma 2.2. If $W$ is a P-function on $X$, then the $N C P(W)$ has at most one solution.

Proof. See Proposition 3.5.10 [9].

Lemma 2.3. If there exists a vector $y^{\mathrm{ref}} \in X$ such that the set

$$
L_{<}^{\prime}=\left\{y \in X: W_{i}(y)\left(y_{i}-y_{i}^{\text {ref }}\right)<0 \text { if } y_{i} \neq y_{i}^{\text {ref }} \text { for all } i=1,2, \ldots, n\right\}
$$

is bounded, then the $N C P(W)$ has a solution.

Proof. See Proposition 3.5.1 [9].

Now we give the proof of Proposition 2.2 as follows.

Proof of Proposition 2.2. Let $y^{\text {ref }}=0$ and

$$
L_{0}^{\prime}=\left\{y \in X: W_{i}(y) y_{i}<0 \quad \text { if } y_{i} \neq 0 \text { for all } i=1,2, \ldots, n\right\} .
$$

From Lemmas 2.1-2.3, if $L_{0}^{\prime}$ is bounded, then Proposition 2.2 holds. Now we prove that $L_{0}^{\prime}$ is bounded. Suppose this is false. Then there exists a sequence $\left\{y^{k} \in L_{0}^{\prime}\right\}$ such that $\left\|y^{k}\right\| \rightarrow \infty$. Define the index set $J$ by $J:=\left\{i \mid\left\{y_{i}^{k}\right\}\right.$ is unbounded, $\left.i=1,2, \ldots, n\right\}$. Then $J \neq \emptyset$ because otherwise the sequence $\left\{y^{k} \in L_{0}^{\prime}\right\}$ is bounded. We suppose that

$$
y_{i}^{k} \rightarrow+\infty \text { as } k \rightarrow \infty \text { for any } i \in J .
$$

Otherwise, we can take a subsequence $\left\{y_{i}^{k_{i}}\right\}$ such that $y_{i}^{k_{i}} \rightarrow+\infty$ as $k_{i} \rightarrow \infty$. For each $k$, let

$$
\bar{y}_{i}^{k}=\left\{\begin{array}{ll}
y_{i}^{k} & \text { if } i \notin J, \\
0 & \text { if } i \in J,
\end{array} \quad i=1,2, \ldots, n .\right.
$$

Clearly the sequence $\left\{\bar{y}^{k}\right\} \subset X$ is bounded and for each $k$,

$$
y_{i}^{k}-\bar{y}_{i}^{k}=\left\{\begin{array}{ll}
0 & \text { if } i \notin J, \\
y_{i}^{k} & \text { if } i \in J,
\end{array} \quad i=1,2, \ldots, n,\right.
$$

and

$$
\left(y_{i}^{k}\right)^{1 / p}-\left(\bar{y}_{i}^{k}\right)^{1 / p}=\left\{\begin{array}{ll}
0 & \text { if } i \notin J, \\
\left(y_{i}^{k}\right)^{1 / p} & \text { if } i \in J,
\end{array} \quad i=1,2, \ldots, n .\right.
$$

For each $k$, since $M$ is a $P_{0}$-matrix, there exists, for the two vectors $\left(y^{k}\right)^{1 / p},\left(\bar{y}^{k}\right)^{1 / p} \in$ $X$, an index $i_{k} \in J$ such that

$$
\left[\left(y_{i_{k}}^{k}\right)^{1 / p}-\left(\bar{y}_{i_{k}}^{k}\right)^{1 / p}\right]\left[M\left(\left(y^{k}\right)^{1 / p}-\left(\bar{y}^{k}\right)^{1 / p}\right)\right]_{i_{k}} \geq 0 .
$$


Since $\bar{y}_{i_{k}}^{k}=0$, we have

$$
y_{i_{k}}^{k}\left[M\left(\left(y^{k}\right)^{1 / p}-\left(\bar{y}^{k}\right)^{1 / p}\right)\right]_{i_{k}} \geq 0 .
$$

Hence,

$$
\begin{aligned}
y_{i_{k}}^{k}\left[W\left(y^{k}\right)-W\left(\bar{y}^{k}\right)\right]_{i_{k}} & =y_{i_{k}}^{k}\left[M\left(\left(y^{k}\right)^{1 / p}-\left(\bar{y}^{k}\right)^{1 / p}\right)\right]_{i_{k}}+B_{i_{k}}\left(y_{i_{k}}^{k}\right)^{2} \\
& \geq B_{i_{k}}\left(y_{i_{k}}^{k}\right)^{2} .
\end{aligned}
$$

Thus, we obtain

$$
y_{i_{k}}^{k} W_{i_{k}}\left(y^{k}\right) \geq B_{i_{k}}\left(y_{i_{k}}^{k}\right)^{2}+y_{i_{k}}^{k} W_{i_{k}}\left(\bar{y}^{k}\right) .
$$

Since $J$ has only a finite number of elements, by taking a subsequence if necessary, we may assume that for each $k, i_{k}=i \in J$. So we have

$$
y_{i}^{k} W_{i}\left(y^{k}\right) \geq B_{i}\left(y_{i}^{k}\right)^{2}+y_{i}^{k} W_{i}\left(\bar{y}^{k}\right) .
$$

Since $\left\{\bar{y}^{k}\right\} \subset X$ is bounded, the sequence $\left\{W_{i}\left(\bar{y}^{k}\right)\right\}$ is bounded. From (2.4), for $i \in J$, $y_{i}^{k} \rightarrow+\infty$ as $k \rightarrow \infty$. Hence, from (2.6), we have

$$
y_{i}^{k} W_{i}\left(y^{k}\right) \geq B_{i}\left(y_{i}^{k}\right)^{2}+y_{i}^{k} W_{i}\left(\bar{y}^{k}\right) \rightarrow+\infty \quad \text { as } k \rightarrow \infty .
$$

This is a contradiction to $y^{k} \in L_{0}^{\prime}$; i.e., $y_{i}^{k} W_{i}\left(y^{k}\right)<0$. In view of this contradiction, we conclude that $L_{0}^{\prime}$ is bounded.

The $\mathrm{NCP}(\mathrm{W})$ can be reformulated into some semismooth equations. Based on these equations, we can develop a superlinearly convergent method for $\mathrm{NCP}(\mathrm{W})$. It is well known that solving the $\mathrm{NCP}(\mathrm{W})$ is equivalent to finding a solution of the Robinson's normal equation

$$
E(z):=W\left(\Pi_{X}(z)\right)+z-\Pi_{X}(z)=0
$$

in the sense that if $z^{*} \in \Re^{n}$ is a solution of (2.7), then $y^{*}:=\Pi_{X}\left(z^{*}\right)$ is a solution of the $\operatorname{NCP}(\mathrm{W})$, and conversely if $y^{*}$ is a solution of the $\operatorname{NCP}(\mathrm{W})$, then $z^{*}:=y^{*}-W\left(y^{*}\right)$ is a solution of (2.7) [17]. Here, for any $z \in \Re^{n}, \Pi_{X}(z)$ is the Euclidean projection of $z$ onto $X$.

Let $\phi$ be the Chen-Harker-Kanzow-Smale (CHKS) smoothing NCP function [13], which is defined as

$$
\phi(t, w)=\frac{\sqrt{w^{2}+4 t^{2}}+w}{2}, \quad(t, w) \in \Re^{2} .
$$

For any $t \neq 0$, by simple computation, we have

$$
\frac{\partial \phi(t, w)}{\partial t}=\frac{2 t}{\sqrt{w^{2}+4 t^{2}}} \quad \text { and } \quad \frac{\partial \phi(t, w)}{\partial w}=\frac{1}{2}+\frac{w}{2 \sqrt{w^{2}+4 t^{2}}} .
$$

Define $\phi(t, z)=\left[\phi\left(t, z_{1}\right), \phi\left(t, z_{2}\right), \ldots, \phi\left(t, z_{n}\right)\right]^{T}$. Then, from [14], we have the following proposition. 
Proposition 2.4. $\phi(t, z)$ has the following properties:

(i) For any given $t \neq 0, \phi(t, z)$ is continuously differentiable.

(ii) $\phi(t, z) \in \operatorname{int} X$ for any given $t>0$.

(iii) $\phi(t, z)$ is a strongly semismooth function on $\Re^{n+1}$.

Define $H: \Re^{n+1} \rightarrow \Re^{n+1}$ by

$$
H(t, z):=\left(\begin{array}{c}
t \\
G(t, z)
\end{array}\right)
$$

where

$$
G(t, z)=W(\phi(t, z))+z-\phi(t, z) .
$$

Then $H$ is an augmented smoothing function of $E$ defined in (2.7). Clearly, $H$ is a locally Lipschitzian function, and $H$ is continuously differentiable at any $(t, z) \in \Re^{n+1}$ with $t \neq 0$. From Proposition 2.4 (iii), $\phi(t, z)$ is a strongly semismooth function. Hence, $W$ is a strongly semismooth function as well. Since $H$ is a composite function of the two strongly semismooth functions $W$ and $\phi$, by Theorem 19 [10], we have the following proposition.

PROPOSITION 2.5. $H$ is a strongly semismooth function on $\Re^{n+1}$. Moreover, $H\left(t^{*}, z^{*}\right)=0$ if and only if $t^{*}=0$ and $E\left(z^{*}\right)=0$.

From Proposition 2.5, solving the system of nonlinear equations (2.7) is equivalent to finding a solution of $H(t, z)=0$. Hence, we have the following proposition.

Proposition 2.6. Suppose that $\left(t^{*}, z^{*}\right)$ is a solution of the system $H(t, z)=0$. Then $y^{*}=\Pi_{X}\left(z^{*}\right)$ is a solution of the $N C P(W)$, and $x^{*}=\left(\Pi_{X}\left(z^{*}\right)\right)^{\frac{1}{p}}$ is a solution of the $\operatorname{NCP}(F)$.

Let

$$
\begin{aligned}
D_{1} & =\left[\frac{1}{p}\left(\phi\left(t, z_{i}\right)\right)^{\frac{1}{p}-1} \partial \phi\left(t, z_{i}\right) / \partial t, i=1,2, \ldots, n\right]^{T}, \\
D_{2} & =\left[\partial \phi\left(t, z_{i}\right) / \partial t, i=1,2, \ldots, n\right]^{T}, \\
D_{3} & =\operatorname{diag}\left(\frac{1}{p}\left(\phi\left(t, z_{i}\right)\right)^{\frac{1}{p}-1} \partial \phi\left(t, z_{i}\right) / \partial z_{i}, i=1,2, \ldots, n\right), \\
D_{4} & =\operatorname{diag}\left(\partial \phi\left(t, z_{i}\right) / \partial z_{i}, i=1,2, \ldots, n\right) .
\end{aligned}
$$

For any $t \neq 0$, by simple computation, we have

$$
H^{\prime}(t, z):=\left(\begin{array}{cc}
1 & 0 \\
G_{t}^{\prime}(t, z) & G_{z}^{\prime}(t, z)
\end{array}\right)
$$

where

$$
G_{t}^{\prime}(t, z)=M D_{1}+B D_{2}-D_{2}
$$

and

$$
G_{z}^{\prime}(t, z)=M D_{3}+B D_{4}+I_{n}-D_{4} .
$$

Here, $I_{n}$ is the $n \times n$ identity matrix.

Lemma 2.4 (see [16]). Suppose that an $n \times n$ matrix $A$ is a $P_{0}$-matrix and $C$ and $O$ are two diagonal matrices satisfying $C_{i} O_{i} \geq 0$ and $O_{i} \neq 0$ for each $i=1,2, \ldots, n$. Then, $A C+O$ is nonsingular. 
Proposition 2.7. For any $(t, z) \in \Re^{n+1}$ with $t \neq 0$, if $M$ is a $P_{0}$-matrix, then $H^{\prime}(t, z)$ is nonsingular.

Proof. For any $(t, z) \in \Re^{n+1}$ with $t \neq 0$, from (2.8) and (2.9), we have

$$
\phi\left(t, z_{i}\right)>0 \text { and } \partial \phi\left(t, z_{i}\right) / \partial z_{i} \in(0,1), \quad i=1,2, \ldots, n .
$$

By Lemma 2.4, if $M$ is a $P_{0}$-matrix, then $G_{z}^{\prime}(t, z)$ is nonsingular. So $H^{\prime}(t, z)$ is nonsingular.

3. A superlinearly convergent algorithm. In this section, we present a Newton-type method to solve the system $H(t, z)=0$, where $H$ is defined in (2.10). We show that this method has global and superlinear convergence properties if $M$ is a $P_{0}$-matrix. Using this method, we can generate a sequence $\left\{x^{k}\right\} \subset X$ such that $x^{k}$ converges to the unique solution $x^{*}$ of the $\mathrm{NCP}(\mathrm{F})$ if $M$ is a $P_{0}$-matrix.

Choose $\bar{t} \in \Re_{++}$and $\gamma \in(0,1)$ such that $\gamma \bar{t}<0.5$. Define the merit function $\psi: \Re^{n+1} \rightarrow \Re_{+}$by

$$
\psi(t, z):=\|H(t, z)\|^{2}
$$

and define $\beta: \Re^{n+1} \rightarrow \Re_{+}$by

$$
\beta(t, z):=\gamma \min \{1, \psi(t, z)\} .
$$

Our proposed algorithm is stated as follows.

Algorithm 3.1.

Step 0. Choose constants $\delta \in(0,1)$ and $\sigma \in\left(0, \frac{1}{2}\right)$. Let $t^{0}:=\bar{t}, z^{0} \in \Re^{n}$ be an arbitrary point and $k:=0$.

Step 1. If $H\left(t^{k}, z^{k}\right)=0$ then stop. Otherwise, let $\beta_{k}:=\beta\left(t^{k}, z^{k}\right)$.

Step 2. Let

$$
\Delta t^{k}=-t^{k}+\beta_{k} \bar{t}
$$

Compute $\Delta z^{k}$ by solving the following linear system of equations:

(3.1) $G_{z}^{\prime}\left(t^{k}, z^{k}\right) \Delta z^{k}=-G\left(t^{k}, z^{k}\right)-G_{t}^{\prime}\left(t^{k}, z^{k}\right) \Delta t^{k}+R_{k}, \quad\left\|R_{k}\right\| \leq \beta_{k} \bar{t}$.

Step 3. Let $l_{k}$ be the smallest nonnegative integer $l$ satisfying

$$
\psi\left(t^{k}+\delta^{l} \Delta t^{k}, z^{k}+\delta^{l} \Delta z^{k}\right) \leq\left[1-2 \sigma(1-2 \gamma \bar{t}) \delta^{l}\right] \psi\left(t^{k}, z^{k}\right) .
$$

Define $t^{k+1}:=t^{k}+\delta^{l_{k}} \Delta t^{k}$ and $z^{k+1}:=z^{k}+\delta^{l_{k}} \Delta z^{k}$.

Step 4. Replace $k$ by $k+1$ and go to Step 1 .

Remark 1. (a) Algorithm 3.1 can be regarded as an inexact version of the smoothing Newton methods proposed in [14]. In [14], $\Delta z^{k}$ is an exact solution of the following linear system of equations:

$$
G_{z}^{\prime}\left(t^{k}, z^{k}\right) \Delta z^{k}=-G\left(t^{k}, z^{k}\right)-G_{t}^{\prime}\left(t^{k}, z^{k}\right) \Delta t^{k} .
$$

In our proposed method, we require the linear system (3.3) to be solved inexactly. When the linear system (3.3) is large, the computation of an exact solution requires a lot of computer time and memory. In such a situation, one is forced to compute only an approximate solution by an iterative method.

More recently, in a paper by Gao and Sun [11], an inexact smoothing Newton method has been proposed for solving the least squares covariance matrix problem. 
The numerical results reported in [11] show this method is very efficient for solving the least squares covariance matrix problem with simple constraints.

(b) Let $d^{k}=\left[\Delta t^{k},\left(\Delta z^{k}\right)^{T}\right]^{T} \in \Re^{n+1}$ and $r^{k}=\left[\beta_{k} \bar{t}, R_{k}^{T}\right]^{T} \in \Re^{n+1}$. From Step 2 of Algorithm 3.1, we have

$$
H\left(t^{k}, z^{k}\right)+H^{\prime}\left(t^{k}, z^{k}\right) d^{k}=r^{k} \text { and }\left\|r^{k}\right\| \leq 2 \beta_{k} \bar{t} .
$$

In [14], $d^{k}$ is obtained by solving the following system exactly:

$$
H\left(t^{k}, z^{k}\right)+H^{\prime}\left(t^{k}, z^{k}\right) d^{k}=r_{e}^{k}
$$

where $r_{e}^{k}=\left[\beta_{k} \bar{t}, 0\right]^{T} \in \Re^{n+1}$ and $\left\|r_{e}^{k}\right\|=\beta_{k} \bar{t}$.

(c) Algorithm 3.1 has global and superlinear properties under the condition that $M$ is a $P_{0}$-matrix. For Example 1, the assumption that $M$ is a $P_{0}$-matrix can be guaranteed by a proper discretization method such as the central difference, the piecewise linear finite element, or a finite volume method. See [18].

In the following, we will give the convergence results for our proposed method. Note that Lemmas 3.1-3.3 are modifications of the corresponding results in [14].

Lemma 3.1. Suppose that for some $(\tilde{t}, \tilde{z}) \in \Re_{++} \times \Re^{n}, H^{\prime}(\tilde{t}, \tilde{z})$ is nonsingular. Then, there exist a closed neighborhood $\mathcal{N}(\tilde{t}, \tilde{z})$ of $(\tilde{t}, \tilde{z})$ and a positive number $\bar{\alpha} \in$ $(0,1]$ such that for any $(t, z) \in \mathcal{N}(\tilde{t}, \tilde{z})$ and all $\alpha \in[0, \bar{\alpha}]$, it holds that $t \in \Re_{++}$, $H^{\prime}(t, z)$ is invertible, and

$$
\psi(t+\alpha \Delta t, z+\alpha \Delta z) \leq[1-2 \sigma(1-2 \gamma \bar{t}) \alpha] \psi(t, z),
$$

where $\Delta t=-t+\beta(t, z) \bar{t}$ and $\Delta z$ is a solution of the following linear system of equations:

$$
G_{z}^{\prime}(t, z) \Delta z=-G(t, z)-G_{t}^{\prime}(t, z) \Delta t+R, \quad\|R\| \leq \beta(t, z) \bar{t} .
$$

Proof. Since $H^{\prime}(\tilde{t}, \tilde{z})$ is invertible and $\tilde{t} \in \Re_{++}$, there exists a closed neighborhood $\mathcal{N}(\tilde{t}, \tilde{z})$ of $(\tilde{t}, \tilde{z})$ such that for any $(t, z) \in \mathcal{N}(\tilde{t}, \tilde{z})$, we have $t \in \Re_{++}^{n}$, and $H^{\prime}(t, z)$ is invertible. For any $(t, z) \in \mathcal{N}(\tilde{t}, \tilde{z})$, let $\Delta t=-t+\beta(t, z) \bar{t}$, and let $\Delta z$ be a solution of the following linear system of equations:

$$
G_{z}^{\prime}(t, z) \Delta z=-G(t, z)-G_{t}^{\prime}(t, z) \Delta t+R, \quad\|R\| \leq \beta(t, z) \bar{t} .
$$

Let $d=\left[\Delta t,(\Delta z)^{T}\right]^{T} \in \Re^{n+1}$ and $r=\left[\beta(t, z) \bar{t}, R^{T}\right]^{T} \in \Re^{n+1}$. Then, we have

$$
H(t, z)+H^{\prime}(t, z) d=r, \quad\|r\| \leq 2 \beta(t, z) \bar{t}
$$

For any $\alpha \in[0,1]$, define

$$
g_{(t, z)}(\alpha)=H(t+\alpha \Delta t, z+\alpha \Delta z)-H(t, z)-\alpha H^{\prime}(t, z) d .
$$

It follows from the mean value theorem that

$$
g_{(t, z)}(\alpha)=\alpha \int_{0}^{1}\left[H^{\prime}(t+\theta \alpha \Delta t, z+\theta \alpha \Delta z)-H^{\prime}(t, z)\right] d d \theta .
$$

Since $H^{\prime}(\cdot)$ is uniformly continuous on $\mathcal{N}(\tilde{t}, \tilde{z})$ and $d \rightarrow \tilde{d}=\left[\Delta \tilde{t},(\Delta \tilde{z})^{T}\right]^{T}$ as $(t, z) \rightarrow$ $(\tilde{t}, \tilde{z})$, it follows that for all $(t, z) \in \mathcal{N}(\tilde{t}, \tilde{z})$,

$$
\lim _{\alpha \downarrow 0}\left\|g_{(t, z)}(\alpha)\right\| / \alpha=0 .
$$


Then, from (3.5), (3.6), and the facts that $\beta(t, z) \leq \gamma[\psi(t, z)]^{\frac{1}{2}}$ and $\|r\| \leq 2 \beta(t, z) \bar{t}$, for all $\alpha \in[0,1]$ and all $(t, z) \in \mathcal{N}(\tilde{t}, \tilde{z})$, we have

$$
\begin{aligned}
\psi( & +\alpha \Delta t, z+\alpha \Delta z) \\
& =\|H(t+\alpha \Delta t, z+\alpha \Delta z)\|^{2} \\
& =\left\|H(t, z)+\alpha H^{\prime}(t, z) d+g_{(t, z)}(\alpha)\right\|^{2} \\
& =\left\|(1-\alpha) H(t, z)+\alpha r+g_{z}(\alpha)\right\|^{2} \\
& \leq(1-\alpha)^{2} \psi(z)+2(1-\alpha) \alpha\|H(t, z)\|\|r\|+o(\alpha)+O\left(\alpha^{2}\right) \\
& \leq(1-\alpha)^{2} \psi(z)+4 \alpha\|H(t, z)\| \beta(t, z) \bar{t}+o(\alpha)+O\left(\alpha^{2}\right) \\
& \leq(1-\alpha)^{2} \psi(z)+4 \alpha \gamma \bar{t} \psi(z)+o(\alpha) \\
& \leq(1-2 \alpha) \psi(z)+4 \alpha \gamma \bar{t} \psi(z)+o(\alpha) \\
& =[1-2(1-2 \gamma \bar{t}) \alpha] \psi(z)+o(\alpha) \\
& \leq[1-2 \rho(1-2 \gamma \bar{t}) \alpha] \psi(z)+o(\alpha) .
\end{aligned}
$$

Thus, by virtue of $(3.7)$, we can find a positive number $\bar{\alpha} \in(0,1]$ such that for all $\alpha \in[0, \bar{\alpha}]$ and all $(t, z) \in \mathcal{N}(\tilde{t}, \tilde{z}),(3.4)$ holds.

Lemma 3.2. Suppose that $M$ is a $P_{0}$-matrix. Then, Algorithm 3.1 is well defined at the $k$ th iteration and for any $k \geq 0$. Furthermore,

$$
0<t^{k+1} \leq t^{k} \leq \bar{t}
$$

and

$$
t^{k} \geq \beta\left(t^{k}, z^{k}\right) \bar{t}
$$

Proof. It follows from Proposition 2.7 and Lemma 3.1 that Algorithm 3.1 is well defined at the $k$ th iteration. By the same argument as that given in the proof of Proposition 16 [14], we have (3.8) and (3.9).

Lemma 3.3. Suppose that $M$ is a $P_{0}$-matrix. Then, an infinite sequence $\left\{\left(t^{k}, z^{k}\right)\right\}$ is generated by Algorithm 3.1, and each accumulation point $(\tilde{t}, \tilde{z})$ of $\left\{\left(t^{k}, z^{k}\right)\right\}$ is a solution of $H(t, z)=0$.

Proof. From Lemma 3.2 and Proposition 2.7, it follows that an infinite sequence $\left\{\left(t^{k}, z^{k}\right)\right\}$ is generated such that $t^{k} \geq \beta_{k} \bar{t}$ for all $k \geq 0$. From Algorithm 3.1, $\psi\left(t^{k+1}, z^{k+1}\right)<\psi\left(t^{k}, z^{k}\right)$ for all $k \geq 0$. Let $\psi_{k}=\psi\left(t^{k}, z^{k}\right)$. Hence, the two sequences $\left\{\psi_{k}\right\}$ and $\left\{\beta_{k}\right\}$ are monotonically decreasing. Since $\psi_{k}, \beta_{k}>0(k \geq 0)$, there exist $\tilde{\psi}, \tilde{\beta} \geq 0$ such that $\psi_{k} \rightarrow \tilde{\psi}$ and $\beta_{k} \rightarrow \tilde{\beta}$ as $k \rightarrow \infty$. If $\tilde{\psi}=0$ and $\left\{\left(t^{k}, z^{k}\right)\right\}$ has an accumulation point $(\tilde{t}, \tilde{z})$, then, from the continuity of $\psi(\cdot)$ and $\beta(\cdot)$, we obtain $\psi(\tilde{t}, \tilde{z})=0$ and $\beta(\tilde{t}, \tilde{z})=0$. Thus, we obtain the desired result. Suppose that $\tilde{\psi}>0$ and $(\tilde{t}, \tilde{z}) \in \Re^{n+1}$ is an accumulation point of $\left\{\left(t^{k}, z^{k}\right)\right\}$. By taking a subsequence, if necessary, we may assume that $\left\{\left(t^{k}, z^{k}\right)\right\}$ converges to $(\tilde{t}, \tilde{z})$. Let $\tilde{\psi}=\psi(\tilde{t}, \tilde{z})$ and $\tilde{\beta}=\beta(\tilde{t}, \tilde{z})$. It is easy to see that $\tilde{t} \geq \tilde{\beta} \bar{t}>0$. Then, from Proposition $2.7, H^{\prime}(\tilde{t}, \tilde{z})$ exists and is invertible. Hence, by Lemma 3.3, there exist a closed neighborhood 
$\mathcal{N}(\tilde{t}, \tilde{z})$ of $(\tilde{t}, \tilde{z})$ and a positive number $\bar{\alpha} \in(0,1]$ such that for any $(t, z) \in \mathcal{N}(\tilde{t}, \tilde{z})$ and all $\alpha \in[0, \bar{\alpha}]$, we have $t \in \Re_{++}, H^{\prime}(t, z)$ is invertible, and

$$
\psi(t+\alpha \Delta t, z+\alpha \Delta z) \leq[1-2 \sigma(1-2 \gamma \bar{t}) \alpha] \psi(t, z)
$$

where $\Delta t=-t+\beta(t, z) \bar{t}$ and $\Delta z$ is a solution of the following linear system of equations:

$$
G_{z}^{\prime}(t, z) \Delta z=-G(t, z)-G_{t}^{\prime}(t, z) \Delta t+R, \quad\|R\| \leq \beta(t, z) \bar{t} .
$$

Therefore, for a nonnegative integer $l$ such that $\delta^{l} \in(0, \bar{\alpha}]$, we have

$$
\psi\left(t^{k}+\delta^{l} \Delta t^{k}, z^{k}+\delta^{l} \Delta z^{k}\right) \leq\left[1-2 \sigma(1-2 \gamma \bar{t}) \delta^{l}\right] \psi\left(t^{k}, z^{k}\right)
$$

for all sufficiently large $k$. Then, for every sufficiently large $k, l_{k} \leq l$ and hence $\delta^{l_{k}} \geq \delta^{l}$. Thus,

$$
\psi\left(t^{k+1}, z^{k+1}\right) \leq\left[1-2 \sigma(1-2 \gamma \bar{t}) \delta^{l_{k}}\right] \psi\left(t^{k}, z^{k}\right) \leq\left[1-2 \sigma(1-2 \gamma \bar{t}) \delta^{l}\right] \psi\left(t^{k}, z^{k}\right)
$$

for all sufficiently large $k$. This contradicts the fact that the sequence $\left\{\psi_{k}\right\}$ converges to $\tilde{\psi}>0$. So, we complete the proof.

Proposition 3.1. Suppose that $M$ is a $P_{0}$-matrix. Then, the level set

$$
L\left(t^{0}, z^{0}\right)=\left\{(t, z) \in \Re^{n+1} \mid \psi(t, z) \leq \psi\left(t^{0}, z^{0}\right)\right\}
$$

is bounded.

Proof. On the contrary, suppose that there exists a sequence $\left\{\left(t^{k}, z^{k}\right) \in \Re^{n+1}\right\}$ such that $\left(t^{k}, z^{k}\right) \in L\left(t^{0}, z^{0}\right)$ and $\left\|\left(t^{k}, z^{k}\right)\right\| \rightarrow \infty$. Since $\left\{t^{k}\right\}$ is bounded, $\left\|z^{k}\right\| \rightarrow \infty$. It is easy to prove that for $i=1,2, \ldots, n$,

$$
\left|\max \left(0, z_{i}^{k}\right)\right| \rightarrow \infty \Longrightarrow\left|z_{i}^{k}\right| \rightarrow \infty \text { and }\left|z_{i}^{k}-\max \left(0, z_{i}^{k}\right)\right| \rightarrow 0 .
$$

From the definition of $\phi$, we have

$$
\left|\phi\left(t^{k}, z_{i}^{k}\right)-\max \left(0, z_{i}^{k}\right)\right| \leq t^{k}, \quad i=1,2, \ldots, n .
$$

Define the index set $J$ by $J:=\left\{i \mid\left\{\phi\left(t^{k}, z_{i}^{k}\right)\right\}\right.$ is unbounded, $\left.i=1,2, \ldots, n\right\}$. Then, $J \neq \emptyset$, because otherwise $\left\|G\left(t^{k}, z^{k}\right)\right\|=\left\|W\left(\phi\left(t^{k}, z^{k}\right)\right)+z^{k}-\phi\left(t^{k}, z^{k}\right)\right\| \rightarrow \infty$. For each $k$, let

$$
\bar{\phi}_{i}^{k}=\left\{\begin{array}{ll}
\phi\left(t^{k}, z_{i}^{k}\right) & \text { if } i \notin J, \\
0 & \text { if } i \in J,
\end{array} \quad i=1,2, \ldots, n\right.
$$

Let $\bar{\phi}^{k}=\left[\bar{\phi}_{i}^{k}, i=1,2, \ldots, n\right]^{T}$. Then, $\left\{\left\|\bar{\phi}^{k}\right\|\right\}$ is bounded. Since $M$ is a $P_{0}$-matrix, for each $k$, there exists an $i_{k} \in J$ such that

$$
\left[\left(\phi\left(t^{k}, z_{i_{k}}^{k}\right)\right)^{1 / p}-\left(\bar{\phi}_{i_{k}}^{k}\right)^{1 / p}\right]\left[M\left(\phi\left(t^{k}, z^{k}\right)\right)^{1 / p}-M\left(\bar{\phi}^{k}\right)^{1 / p}\right]_{i_{k}} \geq 0
$$

Because $\bar{\phi}_{i_{k}}^{k}=0$, we have

$$
\phi\left(t^{k}, z_{i_{k}}^{k}\right)\left[M\left(\phi\left(t^{k}, z^{k}\right)\right)^{1 / p}-M\left(\bar{\phi}^{k}\right)^{1 / p}\right]_{i_{k}} \geq 0
$$


Therefore,

$$
\begin{aligned}
& \phi\left(t^{k}, z_{i_{k}}^{k}\right)\left[W\left(\phi\left(t^{k}, z^{k}\right)\right)-W\left(\bar{\phi}^{k}\right)\right]_{i_{k}} \\
& \quad=\phi\left(t^{k}, z_{i_{k}}^{k}\right)\left[M\left(\phi\left(t^{k}, z^{k}\right)\right)^{\frac{1}{p}}-M\left(\bar{\phi}^{k}\right)^{\frac{1}{p}}\right]_{i_{k}}+B_{i_{k}}\left[\phi\left(t^{k}, z_{i_{k}}^{k}\right)\right]^{2} \\
& \quad \geq B_{i_{k}}\left[\phi\left(t^{k}, z_{i_{k}}^{k}\right)\right]^{2} .
\end{aligned}
$$

So,

$$
\left[W\left(\phi\left(t^{k}, z^{k}\right)\right)-W\left(\bar{\phi}^{k}\right)\right]_{i_{k}} \geq B_{i_{k}} \phi\left(t^{k}, z_{i_{k}}^{k}\right) .
$$

Since $B_{i_{k}}>0$ and $\phi\left(t^{k}, z_{i_{k}}^{k}\right) \rightarrow \infty$ as $k \rightarrow \infty$, we have $\left[W\left(\phi\left(t^{k}, z^{k}\right)\right)-W\left(\bar{\phi}^{k}\right)\right]_{i_{k}} \rightarrow$ $\infty$ as $k \rightarrow \infty$. Note that $\left\{\left\|W\left(\bar{\phi}^{k}\right)\right\|\right\}$ is bounded. It follows that for each $k$, there exists at least one $i_{k} \in J$ such that

$$
\left|W_{i_{k}}\left(\phi\left(t^{k}, z^{k}\right)\right)\right| \rightarrow \infty .
$$

Since $J$ has only a finite number of elements, by taking a subsequence if necessary, we may assume that there exists an $i \in J$ such that

$$
\left|W_{i}\left(\phi\left(t^{k}, z^{k}\right)\right)\right| \rightarrow \infty
$$

Thus, by (3.11) and the definition of $J$, there exists at least one $i \in J$ such that

$$
\left|W_{i}\left(\phi\left(t^{k}, z^{k}\right)\right)\right|,\left|\phi\left(t^{k}, z_{i}^{k}\right)\right|,\left|\max \left(0, z_{i}^{k}\right)\right| \rightarrow \infty .
$$

Hence, by (3.10), (3.11), and the boundedness of $\left\{t^{k}\right\}$, it follows that for such $i \in J$, $\left|z_{i}^{k}-\phi\left(t^{k}, z_{i}^{k}\right)\right|$ is bounded. Then, it is clear that for such an $i \in J,\left\{\left|G_{i}\left(t^{k}, z^{k}\right)\right|\right\}$ is unbounded. Because $\left\|H\left(t^{k}, z^{k}\right)\right\| \geq\left|G_{i}\left(t^{k}, z^{k}\right)\right|,\left\{\left\|H\left(t^{k}, z^{k}\right)\right\|\right\}$ is unbounded. This is a contradiction which shows $L\left(t^{0}, z^{0}\right)$ is bounded.

From Proposition 3.1 and Lemma 3.3, we have the following theorem.

Theorem 3.1. Suppose that $M$ is a $P_{0}$-matrix. Then an infinite sequence $\left\{\left(t^{k}, z^{k}\right)\right\}$ is generated by Algorithm 3.1, and there exists an accumulation point $(\tilde{t}, \tilde{z})$ of $\left\{\left(t^{k}, z^{k}\right)\right\}$ such that $(\tilde{t}, \tilde{z})$ is a solution of $H(t, z)=0$.

Define

$$
A\left(0, z^{*}\right)=\left\{\lim H^{\prime}\left(t^{k}, z^{k}\right): t^{k} \downarrow 0^{+} \text {and } z^{k} \rightarrow z^{*}\right\} .
$$

Clearly, by the definition given in $(1.3), A\left(0, z^{*}\right) \subseteq \partial_{B} H\left(0, z^{*}\right)$.

Lemma 3.4. Suppose that all $V \in A\left(0, z^{*}\right)$ are nonsingular. Then, there is a neighborhood $N\left(0, z^{*}\right)$ of $\left(0, z^{*}\right)$ and a constant $C$ such that for any $(t, z) \in N\left(0, z^{*}\right)$ with $t \neq 0, H^{\prime}(t, z)$ is nonsingular and

$$
\left\|\left(H^{\prime}(t, z)\right)^{-1}\right\| \leq C .
$$

Proof. If the conclusion is not true, then there is a sequence $\left\{\left(t^{k}, z^{k}\right)\right\}$ with all $t^{k} \neq 0$ such that $\left(t^{k}, z^{k}\right) \rightarrow\left(0, z^{*}\right)$, and either all $H^{\prime}\left(t^{k}, z^{k}\right)$ are singular or $\left\|\left(H^{\prime}\left(t^{k}, z^{k}\right)\right)^{-1}\right\| \rightarrow+\infty$. Since $H$ is locally Lipschitzian, $\partial H$ is bounded in a neighborhood of $\left(0, z^{*}\right)$. By passing to a subsequence, if necessary, we may assume that $H^{\prime}\left(t^{k}, z^{k}\right) \rightarrow V$. Thus, $V$ must be singular. This is a contradiction to the assumption of the lemma. This completes the proof.

Proposition 3.2. If $M$ is a $P_{0}$-matrix, then all $V \in A\left(0, z^{*}\right)$ are nonsingular. 
Proof. For any $\left(t^{k}, z^{k}\right) \in \Re^{n+1}$ with $t^{k} \neq 0$, from (2.8) and (2.9), we have

$$
\phi\left(t^{k}, z_{i}^{k}\right)>0 \quad \text { and } \quad \partial \phi\left(t^{k}, z_{i}^{k}\right) / \partial z_{i}^{k} \in(0,1), i=1,2, \ldots, n .
$$

Thus, for any $V \in A\left(0, z^{*}\right), V$ can be written as

$$
V=\left(\begin{array}{cc}
1 & 0 \\
G_{t}^{\prime}\left(0, z^{*}\right) & G_{z}^{\prime}\left(0, z^{*}\right)
\end{array}\right)
$$

where

$$
G_{t}^{\prime}\left(0, z^{*}\right)=M D_{1}^{*}+\left(B-I_{n}\right) D_{2}^{*}, \quad G_{z}^{\prime}\left(0, z^{*}\right)=M D_{3}^{*}+B D_{4}^{*}+I_{n}-D_{4}^{*},
$$

and

$$
\begin{aligned}
& D_{1}=\left[\frac{1}{p}\left|z_{i}^{*}\right|^{\frac{1}{p}-1} d_{i}^{*}, i=1, \ldots, n\right]^{T}, \\
& D_{2}=\left[d_{i}^{*}, i=1, \ldots, n\right]^{T}, \quad d_{i}^{*} \in[0,1], \quad i=1, \ldots, n, \\
& D_{3}=\operatorname{diag}\left(\frac{1}{p}\left|z_{i}^{*}\right|^{\frac{1}{p}-1} e_{i}^{*}, i=1, \ldots, n\right), \\
& D_{4}=\operatorname{diag}\left(e_{i}^{*}, \quad i=1, \ldots, n\right), \quad e_{i}^{*} \in[0,1], \quad i=1, \ldots, n .
\end{aligned}
$$

By Lemma 2.4 , if $M$ is a $P_{0}$-matrix, then $G_{z}^{\prime}\left(0, z^{*}\right)$ is nonsingular. Thus, $V$ is nonsingular. This completes the proof.

TheOrem 3.2. Suppose that $M$ is a $P_{0}$-matrix and $\left(0, z^{*}\right)$ is an accumulation point of the infinite sequence $\left\{\left(t^{k}, z^{k}\right)\right\}$ generated by Algorithm 3.1. Then, the whole sequence $\left\{z^{k}\right\}$ converges to $z^{*}$ and the convergence is Q-quadratic, i.e.,

$$
\left\|\left(t^{k+1}, z^{k+1}\right)-\left(0, z^{*}\right)\right\|=O\left(\left\|\left(t^{k}, z^{k}\right)-\left(0, z^{*}\right)\right\|^{2}\right) .
$$

Proof. By Lemma 3.4 and Propositions 2.5 and 3.2, the conclusion of the theorem follows from similar arguments as those given in the proof of Theorem 23 [14].

From Propositions 2.3 and 2.6 and Theorems 3.1 and 3.2, we have the following.

Theorem 3.3. Suppose that $M$ is a $P_{0}$-matrix. Then, an infinite sequence $\left\{\left(t^{k}, z^{k}\right)\right\}$ is generated by Algorithm 3.1. For each $k$, let $x^{k}=\left(\Pi_{X}\left(z^{k}\right)\right)^{\frac{1}{p}}$. Then, the sequence $\left\{x^{k}\right\}$ converges to the unique solution $x^{*}$ of the $N C P(F)$.

4. Numerical experiments. In this section, we report our numerical experiments for testing the efficiency of Algorithm 3.1. At each iteration of Algorithm 3.1, the main task is to solve the linear system (3.1). The matrix $G_{z}^{\prime}\left(t^{k}, z^{k}\right)$ in the system (3.1) is not symmetric, and from (2.13) and Lemma 2.4 it is nonsingular when $M$ is a $P_{0}$-matrix. Therefore, it is natural to choose the BiCGStab [20] solver to solve the linear system (3.1), and from [20,19] this BiCGStab method converges when $G_{z}^{\prime}\left(t^{k}, z^{k}\right)$ is nonsingular.

Algorithm 3.1 was implemented in MATLAB and was run on a PC (Intel 3.20 $\mathrm{GHz}$ with $0.99 \mathrm{~GB}$ of RAM) for the following free boundary problem $[1,4]$.

Example 2. Let $\Omega=(0,1) \times(0,1)$ and $p \in(0,1)$. We consider

$$
\begin{aligned}
-\Delta u+\frac{9}{(1-p)^{2}} u^{p} & =f(z) & & \text { in } \Omega_{+}, \\
u & =0 & & \text { in } \Omega_{0}, \\
u=|\nabla u| & =0 & & \text { on } \Gamma, \\
u & =g(z) & & \text { on } \partial \Omega,
\end{aligned}
$$


where $\Omega_{+}=\{z \in \Omega: u(z)>0\}, \Omega_{0}=\{z \in \Omega: u(z)=0\}$, and $\Gamma=\partial \Omega_{0}=\partial \Omega_{+} \cap \Omega$ are unknown. Let $r^{2}=z_{1}^{2}+z_{2}^{2}$. We choose

$$
f(z)=\frac{9}{r(1-p)^{2}}\left(\frac{3 r-1}{2}\right)^{\frac{2 p}{1-p}} \max \left(0, r-\frac{1}{3}\right)
$$

and

$$
g(z)=\left(\frac{3 r-1}{2}\right)^{\frac{2}{1-p}} \max \left(0, r-\frac{1}{3}\right), \quad z \in \partial \Omega .
$$

Using the five-point finite difference approximation, we obtain an NCP with $F(x)=$ $M x+B x^{p}+q$ for $x \in \Re_{+}^{n}$, and $q$ is a vector in $\Re^{n}$. Here, the components of $x$ are the approximations to the exact solution $u(z)$ at the grid points of $\Omega$, and $M$ is a $H$-matrix.

Throughout the computational experiments, the following parameter values are used:

$$
\bar{t}=0.002, \quad \gamma=0.5, \quad \delta=0.5, \sigma=0.0005, \quad \text { and } z^{0}=0 .
$$

The maximum number of iterations is set as 2000, and the maximum number of the line search is set as 20. At each iteration of Algorithm 3.1, the linear system (3.1) was solved by the bicgstab solver in MATLAB. We stopped the iteration when

$$
\left\|\min \left(x^{k}, F\left(x^{k}\right)\right)\right\|_{\infty} \leq 10^{-7} .
$$

First, we show the efficiency of the bicgstab solver. For Example 2, we use different discretizations $N \in \mathcal{N}$. Note that the dimension of the corresponding complementarity problem is $n=N^{2}$. We report our numerical results in Table 4.1. In this table, Ite denotes the number of iterations of Algorithm 3.1, $\|\min (x, F)\|_{\infty}$ denotes the value of $\left\|\min \left(x^{k}, F\left(x^{k}\right)\right)\right\|_{\infty}$ at the final iteration, and A.N-BiCGStab denotes the average number of the iterations of the bicgstab solver. From this table, we can see the bicgstab solver can solve the linear system (3.1) efficiently and the average number of the iterations is less than $\sqrt{n}=N$, where $n$ is the size of the test problem. This is a nice result for the bicgstab solver.

Next, we will compare our proposed method with the regularized projection method (RPM) proposed in [1] for the non-Lipschitzian NCP (1.1). Throughout the computational experiments, for the RPM, we use

$$
\omega=1, \quad \epsilon_{k}=2^{-k}, \quad k=0,2,4, \ldots, 30 .
$$

For both Algorithm 3.1 and the RPM, the stopping criterion is

$$
\left\|\min \left(x^{k}, F\left(x^{k}\right)\right)\right\|_{\infty} \leq 10^{-7} .
$$

The numerical results obtained are summarized in Tables 4.2 and 4.3. In these two tables, CPU(s) denotes the total computer time in seconds used to solve the problem. For the cases $N=90,120,150$, and 180 , we set $p=0.2,0.3, \ldots, 0.9$. For the cases $N=200,400,600,800$, and 1000 , we let $p=0.6,0.7,0.8,0.9$. Moreover, for the case $N=1000$, the maximum CPU-time set for each algorithm is 10 hours. So "> 10 hours" in Table 4.3 indicates that the algorithm cannot give a solution within 10 hours.

The results reported in Tables 4.2 and 4.3 show that both Algorithm 3.1 and the RPM perform well for these test problems. In particular, we can see that Algorithm 3.1 can produce an approximate solution with high accuracy for these test problems and also can solve very large problems. 
TABLE 4.1

Numerical results of Algorithm 3.1.

\begin{tabular}{|rl|llr|}
\hline \multicolumn{2}{|c|}{ Problem } & \multicolumn{3}{|c|}{ Algorithm 3.1 } \\
\hline $\mathrm{N}$ & $\mathrm{p}$ & Ite & $\|\min (x, F)\|_{\infty}$ & A.N-BiCGStab \\
\hline \hline 10 & 0.70 & 22 & $4.48 \mathrm{e}-008$ & 7.6 \\
10 & 0.90 & 10 & $9.29 \mathrm{e}-008$ & 4.5 \\
\hline 50 & 0.70 & 25 & $5.51 \mathrm{e}-008$ & 44.5 \\
50 & 0.90 & 13 & $2.17 \mathrm{e}-008$ & 21.0 \\
\hline 100 & 0.70 & 25 & $4.92 \mathrm{e}-008$ & 82.2 \\
100 & 0.90 & 13 & $3.82 \mathrm{e}-008$ & 43.8 \\
\hline 300 & 0.70 & 27 & $6.22 \mathrm{e}-008$ & 207.2 \\
300 & 0.90 & 13 & $8.79 \mathrm{e}-008$ & 116.2 \\
\hline 500 & 0.70 & 28 & $7.00 \mathrm{e}-008$ & 330.8 \\
500 & 0.90 & 15 & $5.47 \mathrm{e}-008$ & 183.7 \\
\hline 700 & 0.70 & 29 & $4.43 \mathrm{e}-008$ & 446.4 \\
700 & 0.90 & 14 & $1.76 \mathrm{e}-008$ & 298.0 \\
\hline 900 & 0.70 & 29 & $5.93 \mathrm{e}-008$ & 542.6 \\
900 & 0.90 & 16 & $3.04 \mathrm{e}-008$ & 320.8 \\
\hline
\end{tabular}

TABLE 4.2

Numerical results of Algorithm 3.1 and the RPM.

\begin{tabular}{|rl|llr|ll|}
\hline \multicolumn{2}{|c|}{ Problem } & \multicolumn{3}{|c|}{ Algorithm 3.1} & \multicolumn{2}{c|}{ RPM $[1]$} \\
\hline $\mathrm{N}$ & $\mathrm{p}$ & Ite & $\|$ min $(x, F) \|_{\infty}$ & CPU(s) & $\|\min (x, F)\|_{\infty}$ & $\mathrm{CPU}(\mathrm{s})$ \\
\hline \hline 90 & 0.2 & 462 & $3.53 \mathrm{e}-008$ & 1615.36 & $8.46 \mathrm{e}-008$ & 175.50 \\
90 & 0.3 & 202 & $6.44 \mathrm{e}-008$ & 611.78 & $7.22 \mathrm{e}-008$ & 175.80 \\
90 & 0.4 & 74 & $3.96 \mathrm{e}-008$ & 172.86 & $6.55 \mathrm{e}-008$ & 178.80 \\
90 & 0.5 & 1204 & $9.97 \mathrm{e}-008$ & 2136.56 & $6.16 \mathrm{e}-008$ & 154.80 \\
90 & 0.6 & 36 & $7.82 \mathrm{e}-008$ & 85.80 & $5.99 \mathrm{e}-008$ & 176.58 \\
90 & 0.7 & 25 & $4.45 \mathrm{e}-008$ & 39.83 & $5.96 \mathrm{e}-008$ & 176.42 \\
90 & 0.8 & 17 & $5.45 \mathrm{e}-008$ & 21.36 & $5.96 \mathrm{e}-008$ & 146.56 \\
90 & 0.9 & 13 & $3.62 \mathrm{e}-008$ & 10.00 & $6.44 \mathrm{e}-008$ & 46.45 \\
\hline 120 & 0.2 & 677 & $5.17 \mathrm{e}-008$ & 5448.44 & $\mathbf{5 . 3 0 e}-\mathbf{0 0 5}$ & 513.36 \\
120 & 0.3 & 286 & $9.39 \mathrm{e}-008$ & 2107.55 & $9.88 \mathrm{e}-008$ & 345.02 \\
120 & 0.4 & 86 & $8.64 \mathrm{e}-008$ & 447.50 & $2.83 \mathrm{e}-008$ & 342.73 \\
120 & 0.5 & 174 & $9.93 \mathrm{e}-008$ & 1145.81 & $8.12 \mathrm{e}-008$ & 281.80 \\
120 & 0.6 & 38 & $6.85 \mathrm{e}-008$ & 213.92 & $6.60 \mathrm{e}-008$ & 320.56 \\
120 & 0.7 & 25 & $6.76 \mathrm{e}-008$ & 85.53 & $6.03 \mathrm{e}-008$ & 326.73 \\
120 & 0.8 & 17 & $5.74 \mathrm{e}-008$ & 49.58 & $5.96 \mathrm{e}-008$ & 317.05 \\
120 & 0.9 & 13 & $4.10 \mathrm{e}-008$ & 21.81 & $6.44 \mathrm{e}-008$ & 133.53 \\
\hline 150 & 0.2 & 908 & $5.39 \mathrm{e}-008$ & 14451.89 & $\mathbf{9 . 3 9 e}-\mathbf{0 0 4}$ & 854.31 \\
150 & 0.3 & 416 & $7.43 \mathrm{e}-008$ & 5734.89 & $\mathbf{3 . 5 7 e}-\mathbf{0 0 4}$ & 853.31 \\
150 & 0.4 & 117 & $9.89 \mathrm{e}-008$ & 1304.67 & $\mathbf{4 . 2 8 e}-\mathbf{0 0 5}$ & 855.77 \\
150 & 0.5 & 394 & $9.95 \mathrm{e}-008$ & 4618.00 & $3.48 \mathrm{e}-008$ & 531.94 \\
150 & 0.6 & 37 & $9.43 \mathrm{e}-008$ & 377.16 & $9.56 \mathrm{e}-008$ & 536.20 \\
150 & 0.7 & 26 & $4.33 \mathrm{e}-008$ & 176.50 & $6.63 \mathrm{e}-008$ & 529.78 \\
150 & 0.8 & 17 & $6.12 \mathrm{e}-008$ & 93.56 & $5.99 \mathrm{e}-008$ & 532.02 \\
150 & 0.9 & 13 & $4.38 \mathrm{e}-008$ & 40.58 & $6.45 \mathrm{e}-008$ & 334.05 \\
\hline 180 & 0.2 & 1158 & $3.65 \mathrm{e}-008$ & 32184.38 & $\mathbf{5 . 2 8 e}-\mathbf{0 0 3}$ & 1158.42 \\
180 & 0.3 & 523 & $9.35 \mathrm{e}-008$ & 13253.72 & $\mathbf{3 . 1 5 e}-\mathbf{0 0 3}$ & 1161.84 \\
180 & 0.4 & 142 & $5.66 \mathrm{e}-008$ & 2796.27 & $\mathbf{1 . 1 4 e}-\mathbf{0 0 3}$ & 1190.44 \\
180 & 0.5 & 284 & $9.95 \mathrm{e}-008$ & 6182.42 & $\mathbf{7 . 2 0 e}-\mathbf{0 0 5}$ & 912.89 \\
180 & 0.6 & 38 & $8.73 \mathrm{e}-008$ & 708.69 & $2.98 \mathrm{e}-008$ & 891.89 \\
180 & 0.7 & 26 & $6.71 \mathrm{e}-008$ & 317.06 & $8.64 \mathrm{e}-008$ & 731.09 \\
180 & 0.8 & 17 & $6.41 \mathrm{e}-008$ & 168.64 & $6.18 \mathrm{e}-008$ & 747.06 \\
180 & 0.9 & 13 & $4.80 \mathrm{e}-008$ & 71.17 & $6.46 \mathrm{e}-008$ & 572.41 \\
\hline & & & & & & \\
\hline
\end{tabular}


TABLE 4.3

Numerical results of Algorithm 3.1 and the RPM.

\begin{tabular}{|rr|llr|lr|}
\hline \multicolumn{2}{|c|}{ Problem } & \multicolumn{3}{|c|}{ Algorithm 3.1} & \multicolumn{2}{c|}{ RPM $[1]$} \\
\hline $\mathrm{N}$ & $\mathrm{p}$ & Ite & $\|\min (x, F)\|_{\infty}$ & $\mathrm{CPU}(\mathrm{s})$ & $\|\min (x, F)\|_{\infty}$ & $\mathrm{CPU}(\mathrm{s})$ \\
\hline \hline 200 & 0.6 & 38 & $8.55 \mathrm{e}-008$ & 956.75 & $2.98 \mathrm{e}-008$ & 1410.88 \\
200 & 0.7 & 26 & $8.49 \mathrm{e}-008$ & 463.53 & $3.31 \mathrm{e}-008$ & 985.39 \\
200 & 0.8 & 17 & $6.56 \mathrm{e}-008$ & 245.14 & $6.51 \mathrm{e}-008$ & 929.34 \\
200 & 0.9 & 13 & $5.45 \mathrm{e}-008$ & 95.98 & $6.47 \mathrm{e}-008$ & 771.55 \\
\hline 400 & 0.6 & 42 & $7.52 \mathrm{e}-008$ & 6820.59 & $\mathbf{2 . 7 8 e}-\mathbf{0 0 2}$ & 8170.84 \\
400 & 0.7 & 28 & $4.51 \mathrm{e}-008$ & 3911.89 & $\mathbf{8 . 6 3 e}-\mathbf{0 0 3}$ & 8255.02 \\
400 & 0.8 & 17 & $7.30 \mathrm{e}-008$ & 2075.92 & $2.52 \mathrm{e}-007$ & 8241.44 \\
400 & 0.9 & 14 & $9.65 \mathrm{e}-008$ & 886.25 & $7.48 \mathrm{e}-008$ & 5103.84 \\
\hline 600 & 0.6 & 49 & $9.99 \mathrm{e}-008$ & 19831.94 & $\mathbf{3 . 8 7 e}-\mathbf{0 0 2}$ & 19970.98 \\
600 & 0.7 & 29 & $4.32 \mathrm{e}-008$ & 11135.61 & $\mathbf{3 . 4 1 e}-\mathbf{0 0 2}$ & 19898.55 \\
600 & 0.8 & 17 & $7.61 \mathrm{e}-008$ & 6240.20 & $\mathbf{6 . 6 8 e}-\mathbf{0 0 3}$ & 19962.70 \\
600 & 0.9 & 14 & $4.89 \mathrm{e}-008$ & 3267.95 & $7.39 \mathrm{e}-008$ & 14321.89 \\
\hline 800 & 0.6 & 44 & $9.03 \mathrm{e}-008$ & 28488.84 & $\mathbf{3 . 3 3 e}-\mathbf{0 0 2}$ & 36137.73 \\
800 & 0.7 & 29 & $5.04 \mathrm{e}-008$ & 20648.20 & $\mathbf{3 . 3 9 e}-\mathbf{0 0 2}$ & 36204.59 \\
800 & 0.8 & 17 & $8.87 \mathrm{e}-008$ & 11810.73 & $\mathbf{2 . 5 8 e}-\mathbf{0 0 2}$ & 36222.39 \\
800 & 0.9 & 14 & $6.72 \mathrm{e}-008$ & 7048.72 & $5.00 \mathrm{e}-007$ & 36145.81 \\
\hline 1000 & 0.6 & \multicolumn{3}{|c|}{$>10$ hours } & & \multicolumn{2}{c}{$>10$ hours } \\
1000 & 0.7 & 29 & $7.80 \mathrm{e}-008$ & 32103.77 & $>10$ hours \\
1000 & 0.8 & 18 & $2.79 \mathrm{e}-008$ & 23479.89 & $>10$ hours \\
1000 & 0.9 & 14 & $2.03 \mathrm{e}-008$ & 14017.41 & $>10$ hours \\
\hline
\end{tabular}

5. Conclusions. In this paper, we proposed an inexact smoothing Newton method for solving a class of non-Lipschitzian complementarity problems defined in (1.1). This method has some nice features. (1) It converges quadratically and globally under the condition that $M$ is a $P_{0}$-matrix. This condition is weaker than the ones used in $[1,5]$. (2) Under the condition that $M$ is a $P_{0}$-matrix, the reformulated system $H(t, z)=0$, where $H$ is defined in (2.10), has a unique solution and also enjoys a nonsingularity property. This is vital to apply the BiCGStab iterative solver to get an approximate solution of the resulting linear system (3.1). (3) Numerical results reported in this paper show that this method can produce an approximate solution with high accuracy for large-scale problems.

Acknowledgments. The authors are grateful to the anonymous referees and Professor Defeng Sun for their valuable comments which led to several improvements of the paper. In particular, we thank Professor Sun [19] for some discussions about the BiCGStab solver.

\section{REFERENCES}

[1] G. Alefeld And X. Chen, A regularized projection method for complementarity problems with non-Lipschitzian function, Math. Comp., 77 (2008), pp. 379-395.

[2] L. Angermann and S. Wang, Convergence of a fitted finite volume method for European and American option valuation, Numer. Math., 106 (2007), pp. 1-40.

[3] A. K. AzIZ, A. B. Stephens, And M. SuRI, Numerical methods for reaction-diffusion problems with non-differentiable kinetics, Numer. Math., 53 (1988), pp. 1-11.

[4] J. W. Barrett AND R. M. Shanahan, Finite element approximation of a model reactiondiffusion problem with a non-Lipschitz nonlinearity, Numer. Math., 59 (1991), pp. 217242.

[5] X. CHEN, A superlinearly and globally convergent method for reaction and diffusion problems with a non-Lipschitzian operator, Computing Suppl., 15 (2001), pp. 79-90.

[6] X. Chen, L. QI, And D. Sun, Global and superlinear convergence of the smoothing Newton method and its application to general box constrained variational inequalities, Math. Comp., 67 (1998), pp. 519-540. 
[7] F. H. Clarke, Optimization and Nonsmooth Analysis, Wiley, New York, 1983.

[8] T. De LucA, F. FACChinei, And C. KAnzow, A semismooth equation approach to the solution of nonlinear complementarity problems, Math. Program., 75 (1996), pp. 407-439.

[9] F. Facchinei and J. S. PAng, Finite-Dimensional Variational Inequalities and Complementarity Problems, Springer-Verlag, New York, 2003.

[10] A. Fischer, Solution of monotone complementarity problems with locally Lipschitzian functions, Math. Program., 76 (1997), pp. 513-532.

[11] Y. GAO AND D. Sun, Calibrating least squares covariance matrix problems with equality and inequality constraints, SIAM J. Matrix Anal. Appl., to appear.

[12] A. Greenbaum, Iterative Methods for Solving Linear Systems, SIAM, Philadelphia, 1997.

[13] C. Kanzow, Some noninterior continuation methods for linear complementarity problems, SIAM J. Matrix Anal. Appl., 17 (1996), pp. 851-868.

[14] L. QI, D. Sun, And G. Zhou, A new look at smoothing Newton methods for nonlinear complementarity problems and box constrained variational inequalities, Math. Program., 87 (2000), pp. 1-35.

[15] L. Qi AND J. Sun, A nonsmooth version of Newton's method, Math. Program., 58 (1993), pp. 353-367.

[16] L. QI, D. RAlph, AND G. Zhou, Semiderivative functions and reformulation methods for solving complementarity and variational problems, in Nonlinear Optimization and Related Topics, G. Di Pillo and F. Giannessi, eds., Kluwer, Norwell, MA, 1999, pp. 317-350.

[17] S. M. Robinson, Normal maps induced by linear transformation, Math. Oper. Res., 17 (1992), pp. 691-714.

[18] H. R. Schwarz, Numerical Analysis: A Comprehensive Introduction, Wiley, New York, 1989.

[19] D. Sun, Private communication, 2009.

[20] H. A. Van Der Vorst, Bi-CGSTAB: A fast and smoothly converging variant of Bi-CG for the solution of nonsymmetric linear systems, SIAM J. Sci. Stat. Comput., 13 (1992), pp. 631644.

[21] S. WANG AND C. S. HuAng, A power penalty method for a nonlinear parabolic complementarity problem, Nonlinear Anal., to appear.

[22] S. WANG, X. Q. YAnG, AND K. L. TeO, A power penalty method for a linear complementarity problem arising from American option valuation, J. Optim. Theory Appl., 129 (2006), pp. 227-257. 